\title{
FONOAUDIOLOGIA E APNEIA DO SONO: UMA REVISÃO
}

\author{
Speech therapy and sleepy apnae: a review
}

\author{
Erilucia Pereira Santa Rosa ${ }^{(1)}$, Sandra Maria Alencastro de Oliveira ${ }^{(2)}$, \\ Viviany Andréa Meireles Alves ${ }^{(3)}$, Patrícia Gomes Barboza ${ }^{(4)}$
}

\section{RESUMO}

Tema: a Síndrome da Apneia/Hipopneia Obstrutiva do Sono (SAHOS) é definida pela Academia Americana do Sono como a presença de episódios recorrentes de obstrução parcial ou total das vias aéreas superiores durante o sono e manifesta-se como uma redução (hipopneia) ou cessação completa (apneia) do fluxo aéreo, apesar da manutenção dos esforços inspiratórios. A SAHOS motiva o chamado ronco crônico, sonolência e caracteriza-se pela parada do fluxo aéreo respiratório por pelo menos, 10 segundos. O diagnóstico é realizado através do exame polissonográfico, que consiste no registro simultâneo de atividades do organismo durante a noite, indicando a quantidade de apneias e hipopneias ocorridos e a gravidade da SAHOS. Para sucesso no tratamento desta desordem é fundamental o diagnóstico preciso e correto e a atuação de uma equipe multidisciplinar, estando inserido nela o fonoaudiólogo. Objetivo: analisar, através da literatura a interrrelação da Fonoaudiologia e a SAHOS Conclusão: com o referente estudo, podemos identificar a complexidade da SAHOS e mostrar a importância da atuação fonoaudiológica na terapêutica desses pacientes, para uma melhor qualidade de vida.

DESCRITORES: Síndromes da Apnéia do Sono; Polissonografia; Fonoterapia; Ronco

(1) Aluna do Curso de Graduação em Fonoaudiologia pela Fundação de Ensino Superior de Olinda, FUNESO, Olinda, PE.

(2) Fonoaudióloga; Docente do curso de Fonoaudiologia da Fundação de Ensino Superior de Olinda, FUNESO, Olinda, PE; Docente do curso de Especialização em Voz da Universidade Federal de Pernambuco, UFPE, Recife, PE; Coordenadora da Clinica Escola de Fonoaudiologia da Fundação de Ensino Superior de Olinda, FUNESO, Olinda, PE; Especialista em voz pela Universidade de Franca/ CEFAC - Pós-Graduação em Saúde e Educação; Especialista em Morfologia pela Universidade Federal de Pernambuco; Mestre em Fisiologia pela Universidade Federal de Pernambuco.

(3) Fonoaudióloga; Docente Titular da Universidade Católica de Pernambuco, UNICAP, Recife, PE; e da Fundação de Ensino Superior de Olinda, FUNESO, Olinda, PE; Docente Substituta da Universidade Federal de Pernambuco, UFPE, Recife, PE; Mestrado em Psicologia Cognitiva pela Universidade Federal de Pernambuco; Doutoranda em Nutrição na Universidade Federal de Pernambuco.

(4) Fonoaudióloga da UTI Neonatal do Hospital Agamenon Magalhães, Recife, PE; Fundadora e Responsável pela UTI Neonatal do Hospital Barão de Lucena de Pernambuco, Recife, PE; Mestre em Nutrição pela Universidade Federal de Pernambuco.

Conflito de interesses: inexistente

\section{INTRODUÇÃO}

“...A síndrome da apnéia e hipopnéia obstrutiva do sono é caracterizada por episódios recorrentes de obstrução parcial ou completa interrupção, a despeito do esforço inspiratória ${ }^{1}$...".

A Academia Americana de Medicina do Sono ${ }^{2}$ define a SAHOS como a presença de episódios recorrentes de obstrução parcial ou total das vias aéreas superiores durante o sono e manifesta-se como uma redução (hipopneia) ou cessação completa (apneia) do fluxo aéreo, apesar da manutenção dos esforços inspiratórios. A ausência da ventilação adequada resulta em dessaturação da oxi-hemoglobina que em episódios prolongados ocasiona a hipercapnia e resulta em despertares ocasionando a fragmentação do sono que pode justificar a sonolência excessiva durante o dia, bem como com a hipoxemia recorrente.

Nos últimos anos tem sido crescentes o estudo da SAHOS o seu diagnóstico e suas conse- 
quências. Alterações polissonográficas compatíveis estão presentes em $1 \%$ a $3 \%$ das crianças ${ }^{3}$,e entre os adultos, em $24 \%$ dos homens e $9 \%$ das mulheres. Na faixa etária dos 50 aos 60 anos, tais alterações ocorrerem em $31 \%$ dos homens e $16 \%$ das mulheres ${ }^{4}$.

As consequências da SAHOS em crianças são bem familiares aos otorrinolaringologistas podendo apresentar baixo rendimento escolar, déficit de atenção e hiperatividade, baixa pôndero-estatural e alterações no desenvolvimento do esqueleto facial, com alterações cardiovasculares, em destaque o "cor pulmonale" (forma de insuficiência cardíaca, onde há diminuição da capacidade de funcionamento das câmaras direitas do coração, causada por doença pulmonar).

Nos adultos essas alterações nas áreas neurológicas e respiratórias são mais numerosas podendo incluir redução da memória (com prejuízos na assimilação de novas informações) e déficit na atenção (com maior risco de envolver-se em acidentes, maior irritabilidade e labilidade emocional). Essa síndrome também pode promover alterações inflamatórias, endoteliais, arteroscleróticais, autonômicas e metabólicas, com aumento de risco para hipertensão arterial sistêmica, arritmias cardíacas, doença nas coronárias e insuficiência cardíaca congestiva. Ainda não está claro se há relação entre esta patologia e acidentes vasculares cerebrais ${ }^{4}$.

Ao estudar a SAHOS é necessário o entendimento sobre a respiração e sua relação com essa síndrome. O ronco é um ruído produzido pela vibração da úvula, palato mole, paredes da faringe, epiglote e língua, e alteração da respiração ${ }^{5}$. Os resultados das pesquisas sobre a terapia fonoaudiológica que tem como objetivo adequar a musculatura da orofaringe apresentam resultados satisfatórios já que os resultados referem ter menos despertares noturnos, menos roncos e, durante o dia, houve melhora nas suas atividades diárias ${ }^{6}$.

Esta revisão sobre a SAHOS enfatiza os vários aspectos morfofuncionais e patológicos e a atuação fonoaudiológica junto à equipe multidisciplinar relatando a atuação em prevenção e intervenção o que pode minimizar os sintomas e melhorar a qualidade de vida desses pacientes.

\section{REVISÃO DA LITERATURA}

\section{Conceituação}

Academia Americana de Medicina do Sono ${ }^{1}$ definiu a síndrome da apneia hipopnéia obstrutiva do sono, como episódios recorrentes de obstrução parcial ou total das vias aéreas superiores durante o sono. Manifesta - se como uma redução (hipopneia) ou cessação completa (apneia) do fluxo aéreo. A ausência da ventilação adequada resulta em dessaturação da oxi-hemoglobina que quando prolongadas ocasiona a hipercapnia, terminando com os despertares levando a fragmentação do sono. É um distúrbio crônico, progressivo, que dividir-se em: apneia obstrutiva, onde o fluxo aéreo é impedido pelo colapso das VAS, e em apneia central, quando a ventilação cessa, devido sistema nervoso central ser incapaz de ativar o diafragma e outros músculos respiratórios; em apneias mistas, seguida por aumento sucessivo do esforço respiratório contra a via aérea obstruída ${ }^{7-9}$, resultando em pausas respiratórias por pelo menos 10 segundos e redução transitória e incompleta do fluxo de ar em pelo menos $50 \%$. Esses episódios ocorrem inúmeras vezes durante o sono ${ }^{1,10} \mathrm{e}$ a falta de ventilação prolongada nos alvéolos pulmonares pode promover um aumento progressivo da pressão parcial de gás carbônico no sangue arterial. Esses eventos são interrompidos por microdespertares ${ }^{11}$.

\section{Anatomia do sistema respiratório}

Os órgãos que compõem as vias respiratórias são: nariz, cavidade nasal, faringe, laringe, traqueia e brônquios com suas divisões. A parte superior das vias aéreas é o principal canal para a passagem de ar para os pulmões e estar dividida em quatro subsegmentos anatômicos, que são: nasofaringe, entre as narinas e o palato duro, velofaringe ou orofaringe retropalatal, entre o palato duro e palato mole e a orofaringe que se estende do palato mole até a epiglote e a hipofaringe, que se estende da base da língua até a laringe ${ }^{1}$. Esses segmentos formam a porção colapsável da faringe, os quais são desprovidos de ossos ou cartilagens o que faz com que a sua luz se mantenha pérvia por ação de músculos, os quais ativamente contraem e dilatam o lúmen das vias aéreas superiores ${ }^{12,13}$. As estruturas de tecido mole formam as paredes das vias aéreas superiores, incluindo as amígdalas, palato mole, úvula, língua e parede lateral da faringe. Os tecidos moles e as estruturas ósseas e cartilaginosas aumentam o tecido extraluminal das vias aéreas o que predispõem ao colapso ${ }^{12}$. As estruturas ósseas crânios-faciais que determinam o tamanho da via aérea são: mandíbula e o osso hioide. Nestas os músculos e os tecidos moles se sustentam ${ }^{13}$. Há 24 músculos participando das funções da faringe, ou seja, na deglutição, fala e respiração. O músculo genioglosso tem papel importante na permeabilidade da faringe ${ }^{14}$. No sono a atividade motora do conjunto muscular é diminuída ou abolida, reduzindo a luz do canal e a passagem de ar. Qualquer fator adicional anormal, de redução do calibre da via aérea que se sobreponha a situação fisiológica favorece o colapso durante o sono. 


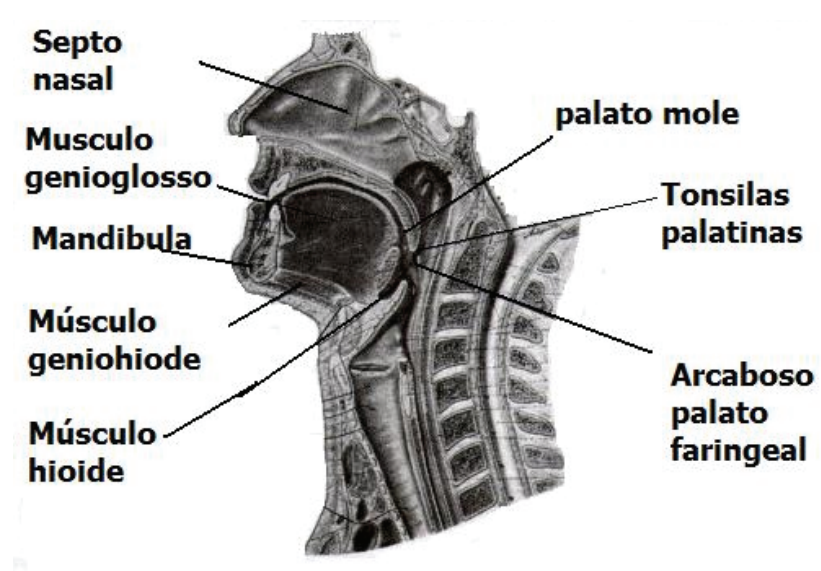

Figura 1 - Esquema da anatomia das vias aéreas superiores, adaptada de Parker, 1997

Na Figura 1 observa-se uma visão sagital das vias aéreas superiores e do pescoço, mostrando a relação entre o palato mole, base da língua, faringe e parede posterior. A literatura revela que pacientes com SAHOS tendem a ter um estreitamento dessas vias, normalmente no final do palato mole e base da língua ${ }^{15}$.

O estreitamento do espaço aéreo faríngeo é causado pela redução das tensões (tonicidade) no músculo genioglosso (língua muscular) e nos músculos que rodeiam a faringe, e a quantidade de tecido que circundam amígdalas, úvula e palato mole ${ }^{15}$. Há um consenso de que o menor calibre das vias aéreas superiores durante a vigília é encontrado na orofaringe retropalatal, o que faz deste ponto um local potencial para seu colapso durante o sono. No entanto, sabe-se que a obstrução pode ocorrer em qualquer dos quatros subsegmentos, de forma simultânea ou sequencial ${ }^{13}$.

$\mathrm{Na}$ faringe ocorre a obstrução nos casos de SAHOS e as evidências demonstram ser essa alteração decorrente de anormalidades anatômicas ou fisiológicas preexistentes ${ }^{16}$. A laringe é uma estrutura tubular flexível e a pressão transmural é quem a mantém aberta. $A$ abertura das vias aéreas superiores depende do balanço entre os músculos dilatadores da faringe e da pressão de sucção intraluminal negativa da mesma durante a inspiração.

\section{Fisiopatologia da SAHOS}

A fisiopatologia da SAHOS embora extensivamente estudada ainda não é completamente elucidada. O fenômeno principal é o colapso da faringe, decorrente, em parte, de alterações morfológicas das vias aéreas superiores, das estruturas faciais e do tônus neuromuscular ${ }^{12,17}$. Dentre as alterações anatômicas, destacam-se a hipertrofia amigdaliana, língua volumosa, pilares amigdalianos medianiza- dos, palato mole posteriorizado e úvula longa e ou espessa ${ }^{18,19}$.

As alterações anatômicas da face, em especial a retrognatia mandibular também está relacionada com a síndrome. Na avaliação facial e oroscopia a oclusão dentária classe II, as alterações das distâncias entre o osso hioide, a tireóide, a cricoíde ${ }^{20,21}$ e a faringe de tamanho reduzido ou com a forma alterada, circular, fazem parte da fisiopatologia da SAHOS.

\section{Epidemiologia}

A prevalência da SAHOS varia de acordo com a idade, sexo, país, metodologia aplicada e do critério empregado no diagnóstico 10,21,22. Os estudos epidemiológicos já realizados demonstram ser alta a prevalência da SAHOS, embora ainda pouco diagnosticada, possivelmente em decorrência da dificuldade de acesso ao diagnóstico e/ou ao alto custo da polissonografia ${ }^{10,23,24}$.

As investigações epidemiológicas populacionais a partir dos anos 90 mostraram que a prevalência da doença é elevada nos adultos, em torno de $23 \%$ a $25 \%$ embora a maioria dos diagnósticos não apresentem comprovações laboratoriais ${ }^{25}$. Em um estudo com voluntários com idade superior a 65 anos, nos paises de Sandiego e Califórnia para diagnosticar a SAHOS revelaram uma prevalência $24 \%{ }^{25,26}$. A prevalência na população foi $5 \%$ acometendo $4 \%$ e $2 \%$ dos homens e mulheres respectivamente ${ }^{27}$. A explicação referida nas pesquisas realizadas para esta predominância é a diferença anatômica existente nas vias aéreas superiores entre homens e mulheres ${ }^{28}$. No Brasil existem referências de prevalência não com base em exames polissonográficos, e sim utilizando dados obtidos em questionário onde estudos no Rio Grande do Sul ${ }^{1}$ relatam prevalência média de $0,9 \%$, entre 1027 trabalhadores industriais. Os resultados indicaram prevalências entre os homens de 1,2\% e de $0,4 \%$ entre as mulheres. Na população infantil, a prevalência foi de $27,6 \%{ }^{29}$ dados significativamente maiores do que os encontrados em outros países, como Alemanha (10\%), Tucson e Europa (10,4\%). Os estudos recentes demonstraram uma tendência no aumento da prevalência da SAHOS em todo mundo quando comparados os resultados e tendências epidemiológicas atuais da obesidade ${ }^{9}$.

\section{Influência do sono na respiração}

A ventilação pulmonar é controlada por um sistema automático, localizado no tronco cerebral e outro voluntário no córtex cerebral. Os quimiorreceptores centrais são sensíveis a variações do $\mathrm{pH}$; o aumento do gás carbônico nesses quimiorreceptores reduz o pH, estimulando-os, enquanto os peri- 
féricos são sensíveis à diminuição da pressão parcial de oxigênio no sangue arterial e do pH. Esses quimiorreceptores estimulam os centros respiratórios localizados no tronco cerebral, controlando a ventilação de forma automática ou metabólica. O controle voluntário é capaz de dominar a função do tronco cerebral, dentro de certos limites. Por exemplo, pode diminuir a $\mathrm{PaCO} 2$ por hiperventilação voluntária ${ }^{1}$. Durante o sono, o único sistema de controle respiratório que atua é o automático. $\mathrm{O}$ sono reduz a sensibilidade dos quimiorreceptores desativa neurônios bulbares e diminui a atividade motora geral, ou seja, ocorre um relaxamento dos músculos incluindo os das VAS (Vias Aéreas Superiores). Esses fenômenos são bem tolerados por pessoas normais, mas em alguns indivíduos podem predispor a distúrbios respiratórios ${ }^{1}$.

Alguns pacientes com SAHOS apresentam hipoxemia crônica, que pode afetar a síntese e a atividade de diversos neurotransmissores, alterando, assim, a função de quimiorreceptores centrais e periféricos responsáveis pelo controle ventilatório. As alterações decorrentes de distúrbios do sono se perpetuam durante a vigília ${ }^{1}$. Pacientes com SAHOS e hipercapnia apresentam uma menor resposta ventilatória à hipercapnia, mesmo durante a vigília. No entanto, quando tratados com pressão positiva contínua nasal demonstram um aumento da resposta ventilatória diurna à hipercapnia e a hipoxemia o que melhora a troca gasosa diurna ${ }^{1}$.

\section{Etiologia}

A SAHOS tem uma etiopatogenia influenciada por múltiplos fatores de risco que estão associados entre si ${ }^{29,30}$. A obesidade e o sobrepeso têm sido considerados um agravante e não um determinante da SAHOS ${ }^{31}$, embora as evidências epidemiológicas apontem a gordura localizada na parte superior do corpo como fator de risco para o seu desenvolvimento ${ }^{9}$.

As condições anatômicas que aumentam a resistência ao fluxo aéreo e predispõem ao colapso da via aérea superior, com o crescimento adenotonsilar é a mais comum ${ }^{32,33}$. No caso de normalidade de via aérea superior e falta de coordenação adequada entre os músculos dilatadores da orofaringe na inspiração existe risco de aparecimento da SAHOS. Durante o sono, há um relaxamento funcional dos músculos faríngeos, responsáveis pela manutenção da via aérea superior ${ }^{32}$. Uma determinada fase do sono é caracterizada pela inibição do tônus dos músculos esqueléticos do organismo, sendo poupados apenas o diafragma e os orbiculares dos olhos. Conforme a hipótese do balanço de forças (pressão barométrica versus tônus muscular faríngeo), a SAHOS ocorre quando há um desequilíbrio que favorece o aumento da pressão negativa na orofaringe durante a inspiração ${ }^{33}$.

Os principais fatores etiológicos são: a hipotonicidade da musculatura que pode estar presente por álcool, drogas, relaxantes, sedentarismo, enveIhecimento, respiração bucal, obesidade, hipertrofia de tonsilas e úvula (por alergia, infecção ou traumatismo), posição em decúbito dorsal, retrognatia e macroglossia ${ }^{7}$. Na infância está envolvida a hipertrofia adenoamigdalinas, doenças neuromusculares como a malformação de Arnold-Chiari III, distrofia miotónica, miopatias e as anomalias craneofaciais como a microretrognatia. Podem contribuir também os fatores inflamatórios, que produzem inflamação local da mucosa da via aérea e obstruem a VAS ${ }^{34}$.

A literatura tem evidenciado que o sexo masculino apresenta maior fator de risco para a SAHOS, mas que na mulher há um aumento da prevalência com a idade principalmente com a proximidade da menopausa pela interferência dos hormônios ${ }^{22,29}$. Há uma relação diretamente proporcional ao aumento de presença da síndrome com o de aumento da probabilidade do índice de massa corporal (IMC), já que esses indivíduos apresentam maiores condições de se apresentarem doenças cardíacas, como a hipertensão arterial, doença arterial coronariana, insuficiência respiratória e cardíaca e acidente vascular cerebral ${ }^{35}$. Os resultados das pesquisas determinam que o sobrepeso e a obesidade aumentam a frequência e gravidade de SAHOS ${ }^{22}$.

\section{Sintomas}

Os portadores de SAHOS apresentam hipersonolência diurna resultante dos microdespertares o que pode aumentar a susceptibilidade diversos acidentes ${ }^{36-38}$. Ao despertar com a parada respiratória, o paciente pode ter dificuldade para retornar o sono, por temer novo evento ${ }^{39}$ e consequentemente haverá aumento de latência e da privação do sono e cochilos diurnos ${ }^{40,41}$.

A SAHOS tipicamente se apresenta na forma de roncos intensos e pausas respiratórias noturnas, de duração variável, comumente por obstrução ao nível da orofaringe que contribui para um sono fragmentado e superficial. Os portadores vivem em estado de continua privação de sono, dificuldades no raciocínio e distúrbios do humor, gerando grande prejuízo pessoal e profissional ${ }^{42}$. A esses sintomas podem associar-se despertares confusionais, às vezes violentos, ou ainda algumas parassonias que são definidas como transtornos que não são anormalidades dos processos responsáveis pelos estados de sono e vigília por si, mas sim problemas orgânicos indesejáveis que ocorrem predominantemente durante o sono. São divididas em distúrbios 
do despertar parcial, distúrbios da transição sonovigília associadas ao sono REM e outras, relacionadas ao despertar como o terror noturno ${ }^{43}$. Nas crianças os sintomas da SAHOS podem se caracterizar pela diminuição do aprendizado e alterações comportamentais ${ }^{44,45}$.

\section{Diagnósticos da SAHOS}

O diagnóstico da SAHOS é baseado na história clínica e na demonstração objetiva do transtorno respiratório durante o sono ${ }^{9}$. A síndrome das apneias obstrutivas do sono envolve respiração e é de grande valia clínica ${ }^{46}$. O exame objetivo tem seus méritos, e inclui a avaliação geral do individuo para detecção de obesidade e da distribuição da gordura corporal. O exame orofacial e das vias aéreas superiores em busca de condição determinante é de fundamental importância para indicação dos fatores de risco ${ }^{47}$, entretanto, são de pouca sensibilidade e especificidade, incapaz de detectar perturbações respiratórias relacionadas com o sono ${ }^{48}$, necessitando-se assim, portanto, de procedimentos laboratoriais específicos. A polissonografia noturna é o procedimento diagnóstico de referência para a SAHOS, pois consiste no registro simultâneo das variáveis neurofisiológicas que permitem estudar o sono e sua correlação com as diferentes variáveis cardiorrespiratórias ${ }^{9}$.

A oximetria noturna é outra técnica extremamente sensível e útil que detecta a redução repetida da saturação do oxigênio do sangue arterial embora nem todos os isentos apneicos e hipopneicos produzem redução significativa da saturação do oxigênio, de forma que uma oximetria normal não exclui a existência de apneia ${ }^{8}$.

A escala de sonolência de Epworth é uma metodologia subjetiva, que tem contribuindo no diagnóstico da SAHOS, por ser, rápida, de baixo custos e simples de ser aplicada ${ }^{49}$, podendo distinguir os graus moderado e leve ${ }^{13,49}$, sendo mais utilizada para acompanhamentos do pacientes, sem no entanto, substituir a polissonografia uma vez que a escala não consegue avaliar todos os graus de severidade ${ }^{50}$.

A análise cefalométrica é outro procedimento que deve ser considerada entre os exames de rotina ${ }^{51}$, pois permite obter informações sobre anormalidades esqueléticas, contribuindo na escolha do tratamento ${ }^{52}$.

A faringometria acústica é outro método válido entre os exames complementares disponíveis, ela define o nível e os parâmetros da estenose, através das relevantes diferenças estatísticas ${ }^{53}$.

\section{Tratamento}

O tratamento da SAHOS tem como objetivo normalizar a ventilação e a oxigenação noturna, eliminar os roncos e a fragmentação do sono. A equipe multidisciplinar é fundamental para que o paciente receba uma abordagem mais ampla durante o processo de avaliação e intervenção terapêutica. Dentre as condutas existentes para o tratamento da SAHOS destacam-se a pressão positiva em vias aérea superiores (CPAP) ${ }^{54,55}$ que é eficaz e, comumente, o método de primeira escolha, as dietas, os aparelhos ortodônticos intra-orais ${ }^{56} \mathrm{e}$ as cirurgias que tem indicação em casos específicos ${ }^{57-59}$ e a terapia fonoaudiológica, recentemente incorporada às intervenções de tratamentos ${ }^{60,5}$.

Os tratamentos clínicos ou cirúrgicos propostos e possíveis resultados podem ser avaliados através das avaliações clínicas e laboratoriais ${ }^{54-57,60}$ pela vídeopolissonografia de seguimento a partir da redução dos microdispertares, melhora da dessaturação de oxihemoglobina, ausência de arritmia cardíaca, ausência ou redução significativa do ronco, melhora no grau de sonolência diurna e grau de satisfação do paciente.

\section{Contribuição da Fonoaudiologia no tratamento}

A fonoaudiologia é mais uma opção de tratamento para a SAHOS e consiste na adequação dos componentes anátomo-morfológico e anátomofuncionais dos órgãos fonoarticulares, que em conseqüência da doença apresentam flacidez. As alterações decorrentes da respiração inadequada ocasionam fraqueza da musculatura orofaríngea que pode reduzir a partir da fonoterapia específica ${ }^{60}$. Com base nas evidências clínicas encontradas ${ }^{60} \mathrm{e}$ no resultado da polissonografia verificouse que, com a utilização de técnicas específicas de fonoterapia em pacientes com SAHOS, mesmo na presença dos fatores agravantes, é possível diminuir o número de apneias e hipopneias durante o sono, chegando a um nível não mais considerado como SAHOS possibilitando uma qualidade de sono tranquilo e reparador e a melhora na qualidade de vida do paciente e a dos seus familiares. $O$ fonoaudiólogo pode intervir nestes casos e possibilitarará na reeducação das funções dos músculos linguais, com alterações desencadeadas, adequar a forma e função dos grupos musculares que interferem no bom desempenho do sistema estomatognático, aumentar a força muscular, promover posturas que favoreçam um funcionamento mais adequado do sistema estomatognáticos bem como de suas funções ${ }^{5}$.

As pesquisas sobre os resultados dos pacientes portadores de SAHOS que foram submetidos a terapia fonoaudiológica para adequação da 
musculatura da orofaringe foram positivas com resultados satisfatórios com redução dos sintomas e melhor qualidade de vida ${ }^{5}$.

Os relatos também foram positivos para os pacientes que apresentaram adequação do tônus e da mobilidade das estruturas do sistema estomagmático, com ênfase à musculatura posterior da língua e do esfíncter velofaríngeo ${ }^{5}$. Diante disso pode-se afirmar dos resultados promissores da fonoaudiologia no tratamento de pacientes portadores da SAHOS, melhorando significativamente os sintomas iniciais de $40 \%$ a $50 \%$ o número de paradas respiratórias, com considerável diminuição do ronco e sonolência diurna ${ }^{5,60}$, melhorando desta maneira a qualidade de vida dos portadores desta síndrome.

Diante dos resultados obtidos com a terapia fonoaudiológica em pacientes portadores da SAHOS comprova-se a importância desta ciência como mais um recurso para o restabelecimento dos portadores da SAHOS. Desta forma, enquanto ciência deve padronizar técnicas eficazes para o tratamento, já que nas pesquisas recentes são ainda poucas as publicações na referida área ${ }^{5,60}$.

As ações fundamentadas em evidências favorecem não apenas na maior adequação dos procedimentos de avaliação e diagnóstico e das intervenções, mas tem importância fundamental na indicação, através da escolha da forma mais precisa, do melhor caminho a ser seguida, na continuação de uma determinada intervenção, ou quais as possíveis mudanças. A tendência atual da prática clínica vem sendo norteada pela corrente chamada "Medicina Baseada em Evidências" (M.B.E), idealizada pelo epidemiologista britânico Archibald.L. Cochrane, desde $1972{ }^{22}$, cujo enfoque é objetivar a ciência médica de uma forma cada vez mais positivista, baseado em dados consistentes de metanálises à disposição para pesquisas, de tal forma que a medicina seja, cada vez mais, olhada e exercida pelos parâmetros cientificamente comprovados de dados advindos de fontes fidedignas. Faz-se necessário, portanto, que o profissional fonoaudiólogo utilize procedimentos sistematizados, validados e mais objetivos de avaliação e tratamento, considerando os dados não apenas de forma qualitativa e sim quantificando as análises. Dessa forma, será possível, a partir dos marcos referenciais fornecidos pelo modelo teórico, que caracterizam os indicadores de cada fase de desenvolvimento, sistematizar parâmetros para identificação e localização dos sujeitos no percurso do processo de construção cognitiva e de linguagem expressiva ${ }^{5}$. Cabe a Fonoaudiologia, resgatar cientificamente sua competência, responsabilizando-se por sua prática clínica, atuando em parceria e colaborando para a eficiência de outros tratamentos, pois se acredita que toda intervenção clínica em motricidade orofacial tende a ter maior grau de sucesso à medida que propõe soluções a curto / médio prazos, práticas, que possibilitem o treino domiciliar de baixo custo e que ofereçam maior automatização e mínimas recidivas.

\section{CONCLUSÃO}

Ao concluir esta pesquisa foi possível identificar a complexidade que rodeia a síndrome da apneia obstrutiva do sono, com amplo espectro de eventos, anátomo-morfolólogicos, fisiopatológicos, etiológicos com repercussões clínicas sistêmicas. A análise deste estudo sugere a terapia miofuncional oral como mais uma alternativa de tratamento para a SAHOS e que a intervenção terapêutica fonoaudiológica na reabilitação dos pacientes portadores da SAHOS é uma realidade crescente e necessária, sendo assim de fundamental importância para o tratamento desses pacientes. 


\section{ABSTRACT}

Background: the Apnea syndrome / Obstructive Sleepy Hypopnea (SOHAS) is define by the American Academy of Sleep with recurrent presence of episodes of partial or total obstruction in the superior airways during sleep, in addition to showing a reduction (hypopnea) or complete stoppage (apnea) of airflow, although there is an ongoing maintenance of inspiratory efforts. SOHAS motivates the so-called sleepy chronic snoring and sleepiness to dress up by the stop of airflow by at least 10 seconds. The diagnosis is carried out through polysomnographic examination, which consists of the simultaneous recording of body activities during the night, indicating the number of occurring apneas and hypopneas and SOHAS severity. For the successful of disorder treatment it is essential to get an accurate diagnosis and correct actions with a multidisciplinary team, where the Speech Therapist is included. Purpose: to analyze, through literature, the interaction of Speech Therapist and SOHAS. Conclusion: with this study, we can identify SOHAS complexity and show the importance of performance in speech therapy for these patients for a better quality of life.

KEYWORDS: Sleepy Apnea Syndromes; Polysomnography; Speech Therapy; Snoring

\section{REFERÊNCIAS}

1. Martins AB, Tufik S, Moura SMGPT. Síndrome da apnéia - hipopnéia obstrutiva do sono. Fisiopatologia. J Bras Pneumol. 2007;21(5/6):33-43.

2. American Academy of Sleep Medicine. Sleep-related breathing disorders in adults: recommendations for syndrome definitions and measurements techniques in clinical research. Sleep. 1999; 22:667-89.

3. Silva VS, Leite JM. Qualidade de vida e distúrbios do sono em crianças: revisão de literatura. Rev Pediatr Ceará. 2005; 6(1):27-36.

4. Cahali MB. Conseqüências da síndrome da apnéia obstrutiva do sono. Rev Bras Otorrinolaringol. 2007 may/mar; 73(3):34-5.

5. Guimarães KCC. Apnéia e ronco. Tratamento miofuncional orofacial. 2009. São Paulo: Pulso; 2009. $96 \mathrm{p}$.

6. Burger RCP, Caixeta EC, Minno DQ de MSD. A relação entre apnéia do sono, ronco e respiração oral. Rev. CEFAC. 2004; 6(3):266-71.

7. Ramos LVT, Furquim LZ. Dica clínica: Aparelho para apnéia obstrutiva do sono. Rev Clin Ortodont Dental Press. 2004; 3(2):21-6.

8. Balbani APS, Weber SAT, Montovani JC. Atualização em síndrome da apnéia obstrutiva do sono na infância. Rev. Bras. Otorrinolaringol. 2005; 71(1):74-80.

9. Pereira A. Síndrome da apnéia obstrutiva do sono. Fisiopatologia, epidemiologia, conseqüências, diagnósticos e tratamentos. Arq Med. 2007; 21(5/6):159-73.
10. Drager LF. Síndrome da apnéia obstrutiva do sono, hipertensão e inflamação. Rev Bras Hipert. 2006; 13(2):144-5.

11. Schechter MS. Section on pediatrics pulmonologists, subcommittee on obstructive sleep apnea syndrome technical report: diagnosis and management of childhood obstructive sleep apnea syndrome. Pediatrics. 2002 Apr; 109(4):69.

12. Patil PS, Schneider H, Schwartz RA, Smith PL. Adult obstructive sleep apnea pathophysiology and diagnosis. Chest. 2007 Jul; 132(1):325-37.

13. Ayappa I, Rapoport DM. The upper airway in sleep: physiology of the pharyns. Sleep Med Rev. 2003; 7(1):9-33.

14. Silva GA, Giacon LAT. Síndrome das apnéias / hipopnéias obstrutivas do sono (SAHOS). Med. 2006 jun; 39(2):185-94.

15. Isono S, Remmer JE, Tanaka A, Sho Y, Sato $\mathrm{J}$, Mishino T. Anatomy of pharynx in patients with obstrucitive sleep apnea and in normal subjects. J Appl Physiol. 1997; 82(2):1319-26.

16. Arens R, Mcdonough JM, Costarino AT, Mahboubi S, Tayag Kier CE, Maislin G. et al. Ressonância magnética da estrutura das vias aéreas superiores de crianças co síndrome da apnéia hipopnéia obstrutiva do sono. Am J Respirat Critical Care Méd. 2001; 164 (1):698-703.

17. Katz ES, D'Ambrosio CM. Pathophysiology of pediatric obstructive sleep apnea. Proc Am Thorac Soc. 2008; 5(2):253-62.

18. Arens R, Marcus CL. Pathophysiology of upper airway obstruction: a developmental perspective. Sleep. 2004 Aug; 27(5):907-1019.

19. Moreno R, Gregório LC, Miranda SL, Marinho FL. Avaliação da síndrome da apnéia/hipopnéia 
grave do sono após tratamento cirúrgico de avanço maxilomandibular. Einstein. 2007; 5(3):255-7.

20. Tsai WH, Remmers JE, Brant R, Flemong W, Davies J, Macarthur C. A decision rule for diagnostic testing in obstructive sleep apnea. Am J Resp Crit Care Med. 2003 mar; 167(10):1427-32.

21. Punjabi MN. The epidemiology of adult obstructive sleep apnea. Proc Am Thorac Soc. 2008; 5(2):132-43.

22. Daltro $\mathrm{CHC}$, Fontes FHO, Santos-Jesus R, Gregório PB, Araújo LMB. Síndrome da apnéia e hipopnéia obstrutiva do sono: Associação como obesidade, gênero e idade. Arq Bras Endocrinol Metabol. 2006; 50(1):74-81.

23. Pedrosa RP, Lorenzi Filho G, Drager LF. Síndrome da apnéia obstrutiva do sono e doença cardiovascular. Rev Med. 2008 abr-jun; 87(2):121-7.

24. Martinez D, Lenz MCS, Menna-Barreto L. Diagnóstico dos transtornos do sono relacionados ao ritmo circadiano. J Bras Pneumol. 2008; 34(3):173-80.

25. Young T, Palta M, Dempsey J, Skatrud J, Weber $S$, Bard S. The occurrence of sleep-disordered breathing among middle-aged adults. $\mathrm{N}$ England $\mathrm{J}$ Med. 1993 Apr; 328(17):1230 -5.

26. Ancoli-Israel S. Epidemiology of sleep disorders. Clin Geriatr Med. 1989 May; 5(2):347-62.

27. Daniel MM, Lorenzi MC, Leite CC, LorenziFilho G. Pharyngeal dimensions in healthy men and women. Clinic. 2007 Feb; 62(1):5-10.

28. Petry C, Pereira UM, Pritrez PMC, Jones MH, Stein RT. Prevalência de sintomas de distúrbios respiratórios do sono em escolares brasileiros. J Pediatr. 2008 mar/apr; 84(2):123-9.

29. Franco RMC, Araújo MFD, Lima JMA, Junior AL. Síndrome de apnéia-hipopneia obstrutiva do sono: avaliação clínica e polissonográfica. Neurobiol. 2008 out/dez; 71(4):19-30.

30. Poyares D, Moraes W. Obesidade e distúrbio respiratório do sono, uma associação de fatores de risco. Arq Bras Endocrinol Metabol. 2007 Oct; 51(7):1029-30.

31. Vgontzas AN, Papanicolaou DA, Bixer EO, Hopper K, Lotsikas A, Lin H, et al. Sleep apnea and daytime sleepiness and fatigue: relation to visceral obesity, insulin resistance, and hipecytckinemic. J Clin Endocrinol Metab. 2000; 85(3):1151-58.

32. Marcus CL. Pathophysiology of chilhood obstructive sleep apnea: current Concerpts. Resp Physiol. 2000 Feb; 119(2-3):143-54.

33. Pereira JCJ, Pessoa JH. Síndrome da apnéia/ hipopnéia do sono: uma visão pediátrica. Rev Paul Pediatr. 2005; 23(4):184-91.

34. Prado OS, Pérez-Yarza G, Ruiz AA, Bergara EO, Aranburu JM, Knorr JIE. Síndrome de apnéia
- bradipnéia obstrutiva do sono. An Pediatr. 2006; 1(2):85-9.

35. Mancini MC, Aloe F, Tavares S. Apnéia do sono em obesos. Arq Bras Endocrinol Metab. 2000; 44(1):81-90.

36. Shamsuzzaman AS, Caples SM, Somers VK. Sleep deprivation and circulatory control. Sleep. 2003 Dec; 26(8):934-6.

37. Desai AV, Ellis E, Vheatley JR, Grunstein RR. Fatal distraction: a case series of fatal fall-sleep Road accidents and their medico legal outcomes. Med J Aust. 2003 Apr; 178(8):396-9.

38. McEvoy RDA. A sleep at the wheel: Who's at risk? Med J Aust. 2003; 178(8):365-6.

39. Edinger JD, Wohlgemuth WK, Radtke RA, Marsh GR, Quillan RE. Does cognitive-behavioral insomnia therapy alter dysfunctional beliefs about sleep? Sleep. 2001; 24(5):591-9.

40. Ayas NT, Fitzgerald JM, Fleetham JA, White DP, Schulzer M, Ryan CF, et al. Cost-effectiveness of continuous positive airway pressure therapy for moderate to severe obstructive sleep apnea/ hipopnéia. Arch Int Med. 2006; 166 (6):977-84.

41. Cistulli PA, Grunstein RR. Medical devices for the diagnosis and treatment of obstructive sleep apnea. Expert Rev Med Devic. 2005; 2(6):749-63.

42. Rodrigues NND, Guiot M, Tavares P. Sobre um caso de síndrome da apnéia obstrutiva de sono simulando narcolepsia. J Pneumol. 1997; 23(5):264-6.

43. Pressman MR, Meyer TJ, Kendrick-Mohamed J, Figueroa WG, Greenspom LW, Peterson D. Night terrors in an adult precipitated by sleep apnea. Sleep. 1995; 18(9):773-5.

44. Gregório PB, Athanazio RA, Bitencourt AGV, Neves FBCS, Terse R, Hora F. Sintomas de síndrome de apnéia - hipopnéia obstrutiva do sono em crianças. J Bras Pneumol. 2008; 34(6):356-61. 45. Mitchell RB. Sleep-disordered breathing in children: are we underestimating the problem? Eur Respir J. 2005; 25(2):216-7.

46. Knort MM, Souza FB, Martinez D. Sindrome das apnéias-hipopnéias obstrutivas do sono: associação com gênero e obesidade e fatores relacionados à sonolência. J Bras Pneumol. 2008;34(7):496-0.

47. Hoffstein V, Szalai JP. Predictive value of clinical features in diagnosing obstructive sleep apnea. Sleep. 1993 Feb; 16(6):118-22.

48. Redline S, Strohl KP. Recognition and consequences of obstructive sleep apnea hypopnea syndrome. Clin Chest Med. 1999; 19(1):1-19.

49. Gus M, Silva DN, Fernandes J, Cunha CP, Sant'anna GD. Escala de sonolência de Epworth em pacientes com diferentes valores na monitorização ambulatorial de pressão arterial. Arq Bras Cardiol. 2002; 70(11):17-20. 
50. Boari L, Cavalcanti CM, Bannwart SRFD, Sofia OB, Dolei JEL. Avaliação da escala de Epworth ou pacientes com a síndrome da apnéia e hipopnéia obstrutiva do sono. Rev Bras Otorrinolaringol. 2004; 70(6):752-6.

51. Salles C, Campos PSF, Andrade NA, Daltro C. Síndrome da apnéia e hipopnéia obstrutiva do sono: análise cefalométrica. Rev Bras Otorrinolaringol. 2005; 71(3):369-72.

52. Zonato Al, Bittencourt FL, Gregório C, Gregório L, Tufik S. Indicação cirúrgica otorrinolaringológica em um ambulatório para pacientes com síndrome da apnéia e hipopnéia obstrutiva do sono. Rev Bras Otorrinolaringol. 2004; 70(1):46-51.

53. Gelardi M, Giudice AM, Del Cassano FCM, Farras AC, Fiorella ML, Cassano PA. Faringometria acústica: correlações clínico-instrumentais nos distúrbios do sono. Rev Bras Otorrinolaringol. 2006 mar/abr; 73(2):257-65.

54. Mcnicholas WT. Follow-up and outcomes of nasal CPAP therapy in patients with sleep apnea syndrome. Monaldi. Arch Chest Dis. 2001 Dec; 56(6):535-9.
55. Silva $A B$, Lopes EA. Distúrbios do sono. Rev Bras Méd. 2001; 58(12):225-35.

56. Almeida MAO, Texeira AOB, Vieira LS, Quintão CCA. Tratamento da síndrome da apnéia e hipopnéia obstrutiva do sono com aparelhos intrabucais. Rev Bras Otorrinolaringol. 2006 set-out; 72(5):699-703. 57. Marinho FL, Zonato Al, Bittencourt LRA, Gregório CL, Gregório LC, Tufik S. Indicação cirúrgica otorrinolaringológica em pacientes com síndrome da apnéia e hipopnéia obstrutiva do sono. Rev Bras Otorrinolaringol. 2004; 70(1):46-51.

58. Bueno JM, Fuster XM, Laguna JG, Bassas C, Martín GR. Avance geniogloso em El tratamiento del síndrome de apnea obstrutiva del sueño. Rev Esp Cirur Oral Maxilofac. 2005; 27(3):162-6.

59. Neto LM, Fava AS, Lopes HC, Stamm A. Estudo epidemiológico das alterações estruturais da cavidade nasal associada à síndrome da apnéia e hipopnéia obstrutiva do sono SAHOS. Rev Bras Otorrinolaringol. 2005 jul-ago; 71(4):464-6.

60. Silva LMP, Aureliano FTS. Motta AR. Atuação fonoaudiológica na síndrome de apnéia e hipopnéia obstrutiva do sono: relato de caso. Rev. CEFAC. 2007; 9(3):490-6.

DOI: 10.1590/S1516-18462010005000055

RECEBIDO EM: 18/10/2009

ACEITO EM: 21/03/2010

Endereço para correspondência:

Erilucia Pereira Santa Rosa

Rua Cosme José dos Santos, 495

Olinda - PE

CEP: $53250-460$

E-mail: lucia.eri@ hotmail.com 